\title{
Phosphodiesterase type 4 inhibition of activated polymorphonuclear leukocytes in a simulated extracorporeal circulation model
}

\author{
Yukio Sato, MD, PhD
}

Yuji Hiramatsu, MD, PhD

Satoshi Homma, MD, PhD

Shyoko Sato

Masataka Onizuka, MD, PhD

Yuzuru Sakakibara, MD, PhD

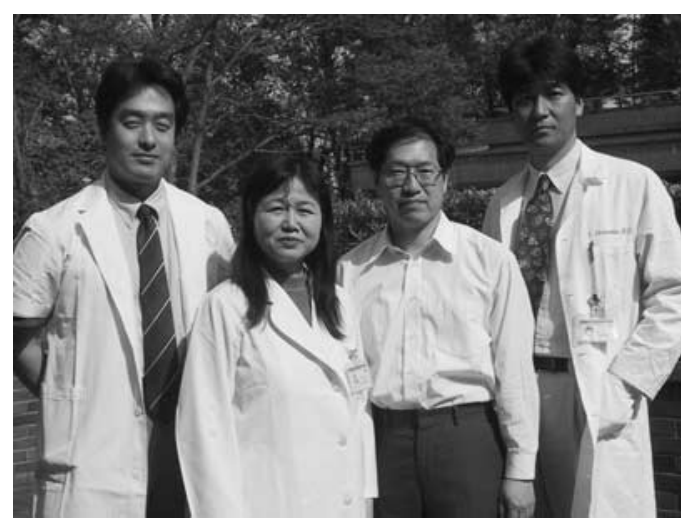

Yukio Sato, Shyoko Sato, Satoshi Homma, Yuji Hiramatsu (left to right)

Objectives: Cardiopulmonary bypass is associated with a systemic inflammatory response syndrome and the risk of multiorgan injuries mediated by activated polymorphonuclear leukocytes. Phosphodiesterase type 4 is the predominant phosphodiesterase isozyme in polymorphonuclear leukocytes and plays a key role in the regulation of polymorphonuclear leukocyte activation. The aim of this study was to examine the effect of rolipram, a selective phosphodiesterase type 4 inhibitor, on the functional changes of polymorphonuclear leukocytes by using simulated extracorporeal circulation.

Methods: Simulated extracorporeal circulation was established by recirculating heparinized human blood for 120 minutes on a membrane oxygenator with and without $10 \mu \mathrm{mol} / \mathrm{L}$ rolipram. F-actin content and L-selectin and CD11b expression of polymorphonuclear leukocytes were measured by means of flow cytometry. Polymorphonuclear leukocyte deformability was evaluated with a microchannel array flow analyzer that had a similar diameter as the capillaries. Polymorphonuclear leukocyte elastase was measured with an enzyme immunoassay.

From the University of Tsukuba, Institute of Clinical Medicine, Tsukuba, Japan.

Supported by University of Tsukuba Research Projects.

Received for publication Feb 5, 2002; revisions requested April 4, 2002; revisions received April 10, 2002; accepted for publication April 16, 2002.

Address for reprints: Yukio Sato, MD., $\mathrm{PhD}$, Division of Thoracic Surgery, Department of Surgery, Jichi Medical School,

3311-1 Minamikawachi, Kawachi, Tochigi, 329-0498 Japan (E-mail: tcvysato@md.jichi.ac.jp).

J Thorac Cardiovasc Surg 2003;125: $172-7$

Copyright (C) 2003 by The American Association for Thoracic Surgery

0022-5223/2003\$30.00+0

doi: $10.1067 / \mathrm{mtc} .2003 .98$
Results: Rolipram reduced the increase of F-actin content of polymorphonuclear leukocytes and the increase of transit time of $100 \mu \mathrm{L}$ of blood sample through a microchannel. Rolipram reduced the increase of $\mathrm{CD} 11 \mathrm{~b}$ expression and the decrease of L-selectin expression of polymorphonuclear leukocytes. Rolipram reduced the release of elastase from polymorphonuclear leukocytes.

Conclusion: Rolipram inhibited the deformability change mediated by F-actin assembly, the changes in adhesion molecules, and the release of elastase from activated polymorphonuclear leukocytes in simulated extracorporeal circulation. This study suggests that phosphodiesterase type 4 inhibition could be a feasible therapeutic strategy to prevent the exaggerated inflammatory response related to cardiopulmonary bypass.

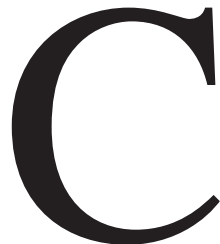

ardiopulmonary bypass (CPB) is associated with a systemic inflammatory response syndrome, and patients who undergo $\mathrm{CPB}$ are at risk of organ injuries typical of acute respiratory distress syndrome. It is well documented that injuries associated with CPB are mediated by activated polymorphonuclear leukocytes (PMNs), ${ }^{1,2}$ and PMN sequestration in microvessels is the initial important step..$^{3,4}$ Activated PMNs sequester in microvessels because of the decrease of their deform- 
ability $^{5}$ and the increase of adhesive qualities of PMNs and endothelial cells. ${ }^{6}$ The decrease in deformability of PMNs is mediated by a rapid assembly of filamentous F-actin from soluble G-actin at the cell periphery. ${ }^{7,8}$ The selectins slow PMNs by mediating rolling, and the integrins induce firm adhesion between PMNs and endothelial cells. ${ }^{6}$ The interaction between these adhesion molecules on PMNs and their ligands on endothelial cells contributes to prolonged PMN sequestration in lung microvessels. ${ }^{9}$

Cyclic adenosine monophosphate (cAMP) is an intracellular messenger that has a suppressant effect on leukocyte activation. ${ }^{10}$ cAMP is inactivated by phosphodiesterase (PDE), and PDE type 4 is the predominant PDE isozyme in leukocytes. ${ }^{10}$ Specific PDE type 4 inhibitors increase cAMP levels in PMNs and are reported to inhibit the increase of cytosolic calcium in $N$-formyl-Met-Leu-Phe-stimulated human PMNs, ${ }^{11}$ which is a pivotal event in PMN activation. ${ }^{12}$

Our working hypothesis is that PDE type 4 inhibitor inhibits the activation and inflammatory response of PMNs during CPB. We used simulated extracorporeal circulation (ECC), which has been used extensively in vitro, to model inflammatory responses during in vivo CPB. ${ }^{13,14}$ The aim of this study was to evaluate the effect of the PDE type 4 inhibitor rolipram on PMN activation by using simulated ECC. We evaluated the effect of rolipram on PMN F-actin assembly, the transit time of whole blood through microchannels, adhesion molecule expression, and the release of elastase from PMNs.

\section{Methods}

\section{Simulated ECC}

The effect of ECC on the activation of PMNs was evaluated with simulated ECC, as described previously in more detail. ${ }^{13,15}$ Each perfusion circuit (surface area of $0.66 \mathrm{~m}^{2}$ ) was assembled from silicone rubber tubing (one-quarter inch inside diameter; MERA co, Tokyo, Japan), polycarbonate connectors, a polyvinyl chloride venous reservoir bag (MERA), and a 0.6- $\mathrm{m}^{2}$ membrane oxygenator (model 60EC, MERA). All components of the circuit were sterilized and used once. Human blood $(250 \mathrm{~mL})$ was drawn from healthy male donors (aged 25-40 years) through an 18-gauge needle and polyvinyl tubing directly into a venous reservoir bag containing heparin $(3.75 \mathrm{U} / \mathrm{mL})$. Donors abstained from all medication for at least 2 weeks before donation. Written informed consent was obtained from donors, and the protocol was approved by the Institutional Review Board of the University of Tsukuba.

The perfusion circuit was filled with blood (control group, $\mathrm{n}=$ 6) or blood containing rolipram (10 $\mu \mathrm{mol} / \mathrm{L}$ final concentration; BIOMOL Research Laboratories, Inc, Plymouth Meeting, Pa; rolipram-treated group, $\mathrm{n}=6$ ). Blood was recirculated by using a barely occlusive roller pump (model MS-033, MERA) at 400 $\mathrm{mL} / \mathrm{min}$ for 120 minutes. Oxygenators were ventilated with $95 \%$ oxygen and $5 \%$ carbon dioxide at a rate of $0.7 \mathrm{~L} / \mathrm{min}$. Blood temperature was maintained at $37^{\circ} \mathrm{C}$ by immersing the reservoir bag in a shaking, constant-temperature water bath. Preliminary experiments confirmed that the $\mathrm{pH}$ of the circulating blood was kept from 7.3 to 7.5 , and the activated clotting time was greater than 500 seconds throughout the experimental period. Four blood samples were drawn from the reservoir into polypropylene tubes before recirculation (zero time) and 30, 60, and 120 minutes after recirculation. The standing control sample $(3.75 \mathrm{U} / \mathrm{mL}$ heparin) was incubated for 120 minutes in a shaking water bath at $37^{\circ} \mathrm{C}$ before processing. Blood samples for blood cell counts and differential white cell counts were collected in tubes containing potassium ethylene tetra-acetic acid (VT-052DK, Venoject; Terumo Medical Corporation, Tokyo, Japan). Blood samples for F-actin, CD11b, L-selectin, and the transit time of whole blood through microchannels were collected into tubes containing acidcitrate-dextrose (9:1 by volume). Blood samples for PMN elastase were collected and centrifuged at $3000 \mathrm{rpm}$ for 15 minutes, and the supernatant was stored at $-80^{\circ} \mathrm{C}$ until measurement.

\section{Blood Cell Counts}

Blood cell counts were performed with a counter (T-660; Beckman Coulter, Inc, Fullerton, Calif), and differential white cell counts were made on Wright-stained blood smears by an experienced independent observer.

\section{F-actin Content Assay}

Fifty microliters of sample was fixed, and the cells were permeabilized with IntraPrep permeabilization reagent (Immunotech, Coulter). PMNs were stained for 30 minutes in the dark at $37^{\circ} \mathrm{C}$ with $1 \mathrm{U}$ of BODIPY FL phallacidin (Molecular Probes Inc, Eugene, Ore). Cells were washed twice with phosphate-buffered saline solution, and F-actin content was measured with a flow cytometer (FACS Calibur, Becton Dickinson, Franklin Lakes, NJ), as previously described, ${ }^{16}$ at a mean fluorescent intensity of 5000 cells. The change of F-actin content was expressed as the percentage change from the baseline value.

\section{Transit of Whole Blood Through Microchannels}

The transit time of whole blood through microchannels was measured as a surrogate marker of PMN deformability by using a microchannel array flow analyzer. The microchannels are made of silicone resembling the size of capillaries (width, $6 \mu \mathrm{m}$; depth, 4.5 $\mu \mathrm{m}$; length, $10 \mu \mathrm{m}$; number of channels, 2600). Whole blood filterability was assessed by measuring the transit time of a purified $100-\mu \mathrm{L}$ sample through the microchannels with a pressure of 10 $\mathrm{cm} \mathrm{H}_{2} \mathrm{O}$, and the passage was recorded with a videomicroscope. ${ }^{17}$

\section{Adhesion Molecules Assay}

Changes in the surface expression of L-selectin and CD11b of PMNs were measured by using flow cytometry, as previously described. ${ }^{16}$ One hundred microliters of whole blood samples was incubated for 30 minutes with $2 \mathrm{mg} / \mathrm{mL}$ fluorescein isothiocyanate-conjugated CD62L antibody (BD Biosciences Pharmingen, San Diego, Calif) and $1 \mathrm{mg} / \mathrm{mL}$ phycoerythrin-conjugated mouse monoclonal anti-human CD11b antibody (DAKO Corporation, Carpinteria, Calif) at $4^{\circ} \mathrm{C}$. Identical samples were incubated with fluorescein isothiocyanate-conjugated mouse immunoglobulin (Ig) $\mathrm{G}$ (DAKO) and phycoerythrin-conjugated mouse IgG2a (DAKO) as a negative control. The erythrocytes were lysed for 60 seconds with Immuno-lyse, and leukocytes were fixed with Immuno-fix (commercial kit from Coulter Clone, Coulter Immuno- 


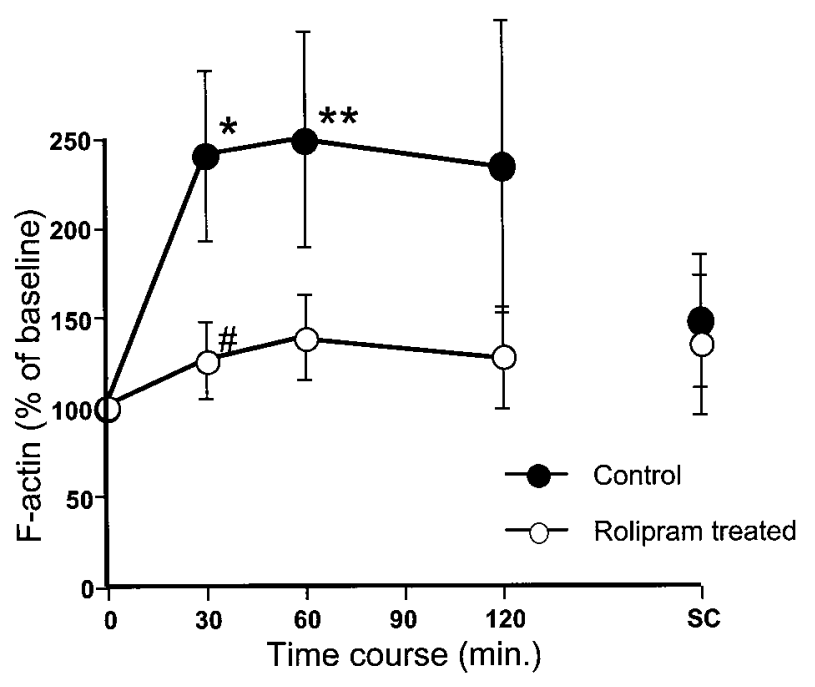

Figure 1. F-actin content of PMNs. The F-actin content of PMNs increased at $\mathbf{3 0}$ and $\mathbf{6 0}$ minutes in the control group. The F-actin content did not increase significantly in the rolipram-treated group. Rolipram attenuated the change in the cytoskelton of PMNs induced by simulated ECC. Results are expressed as means \pm SEM of 6 experiments. ${ }^{*} P=.014,{ }^{* *} P=.032$ versus baseline; $\# P=.042$ versus the control group. $S C$, Standing control.

tech). PMNs were identified by the typical forward- and sidescatter pattern, and the expression of L-selectin and CD11b was measured as a mean fluorescent intensity of 5000 cells of PMNs. The changes of L-selectin and CD11b expression were presented as percentage changes compared with baseline values.

\section{Serum Assays}

PMN elastase was measured with an enzyme-linked immunosorbent assay kit (MERCK Immunoassay, Whitehouse Station, NJ), according to the manufacturer's instructions.

\section{Statistics}

Data were analyzed by using a 2-way analysis of variance in a randomized block design, with time as a repeating factor and donor as a grouping factor. The sequential rejective Bonferroni test was used to correct for multiple comparisons. ${ }^{18}$ All values are expressed as means $\pm \mathrm{SEM}$.

\section{Results}

\section{Blood Cell Counts}

White blood cell counts and hematocrit levels did not change significantly in either group (data not shown). Platelet counts decreased in both the control $(23.0 \% \pm 2.4 \%$ of the baseline value at 120 minutes, $P<.001)$ and rolipramtreated groups $(27.4 \% \pm 3.9 \%$ of the baseline value at 120 minutes, $P<.001)$.

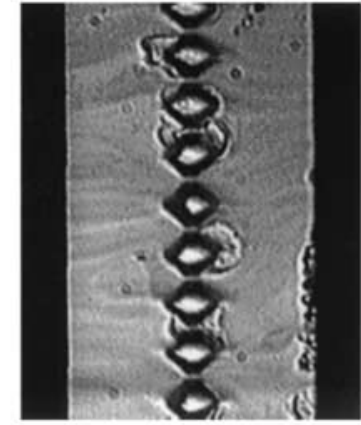

A Control

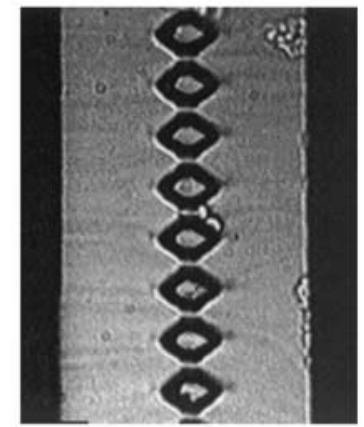

Rolipram treated

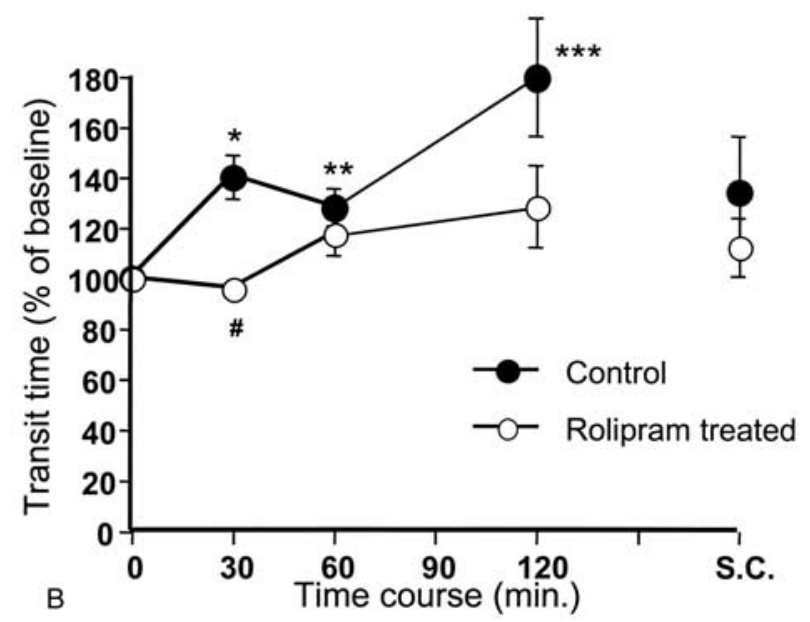

Figure 2. The transit of whole blood through microchannels. A, The videomicroscope image at $\mathbf{1 2 0}$ minutes shows the plugging of microchannels with leukocytes and the disturbance of red blood cell flow in the control group. Rolipram reduced this plugging and kept the flow smooth. B, The transit time of whole blood through microchannels increased from $\mathbf{3 0}$ to $\mathbf{1 2 0}$ minutes in the control group. The transit time did not increase significantly in the rolipram-treated group. Results are expressed as means \pm SEM of 6 experiments. ${ }^{*} P<.001,{ }^{*} P=.001,{ }^{*}{ }^{*} P=.003$ versus baseline; $\# P=.002$ versus the control group. $S C$, Standing control.

\section{F-actin Content Assay}

The F-actin content of PMNs increased to $249.1 \% \pm 60.1 \%$ of the baseline value at 60 minutes in the control group $(P=$ .032 , Figure 1$)$. The F-actin content did not change significantly in the rolipram-treated group $(138.9 \% \pm 23.7 \%$ of the baseline value at 60 minutes). Rolipram inhibited the change in the cytoskelton of PMNs induced by simulated ECC.

Transit of Whole Blood Through Microchannels The videomicroscope image at 120 minutes showed the plugging of microchannels with leukocytes and the disturbance of red blood cell flow in the control group (Figure 2, 
A). Rolipram reduced this plugging and kept the flow smooth. The transit time of whole blood through microchannels increased to $179.8 \% \pm 23.7 \%$ of the baseline value at 120 minutes in the control group $(P<.001$, Figure $2, B)$. The transit time did not change significantly in the rolipram-treated group $(128.5 \% \pm 15.9 \%$ of the baseline value at 120 minutes). Rolipram attenuated the change in whole blood filterability induced by simulated ECC.

\section{Adhesion Molecules Assay}

The expression of L-selectin of PMNs decreased to $17.0 \%$ $\pm 3.6 \%$ of the baseline value at 120 minutes in the control group $(P<.001$; Figure $3, A)$; rolipram attenuated this decrease $(39.2 \% \pm 4.7 \%$ of the baseline value at 120 minutes, $P=.002$ ). The expression of CD11b of PMNs increased to $574.6 \% \pm 50.7 \%$ of the baseline value at 120 minutes in the control group $(P<.001$; Figure $3, B)$; rolipram inhibited this increase $(224.1 \% \pm 67.1 \%$ of the baseline value at 120 minutes, $P=.003$ ). Rolipram inhibited the change in adhesive quality of PMNs induced by simulated ECC.

\section{Serum Assays}

Rolipram reduced the increase of elastase release from PMNs (control group, $1839 \pm 364 \mu \mathrm{g} / \mathrm{mL}$; rolipram-treated group, $801.3 \pm 116.1 \mu \mathrm{g} / \mathrm{mL}$ at 120 minutes; $P=.042$; Figure 4).

\section{Discussion}

Activated PMNs play an important role in the systemic inflammatory response associated with CPB. ${ }^{1,2}$ PMNs are activated by both direct contact with the synthetic surface and the activated complement. ${ }^{2,19,20}$ Our results show that the selective PDE type 4 inhibitor inhibits the activation of PMNs induced by simulated ECC. This was associated with the inhibition of the increase in F-actin content, the decrease in PMN deformability, the decrease in L-selectin expression, the increase in CD11b expression, and the release of elastase from PMNs. These phenotypic and functional characteristics of PMNs have all been shown to affect PMNmediated organ injuries. ${ }^{5}$

Simulatad ECC increased the F-actin content of PMNs at 30 and 60 minutes (Figure 1). The change at 120 minutes was not significant. We speculate that the increase of F-actin might have diminished by 120 minutes in some experiments because the increase of F-actin is a transient phenomenon. Rolipram inhibited this increase in F-actin content of PMNs. The increase in F-actin content was associated with the decrease in PMN deformability, which was measured as the increase in whole blood transit time through microchannels. Rolipram inhibited this decrease in PMN deformability induced by simulated ECC. The microchannel array flow analyzer is a device with uniform silicon channels that
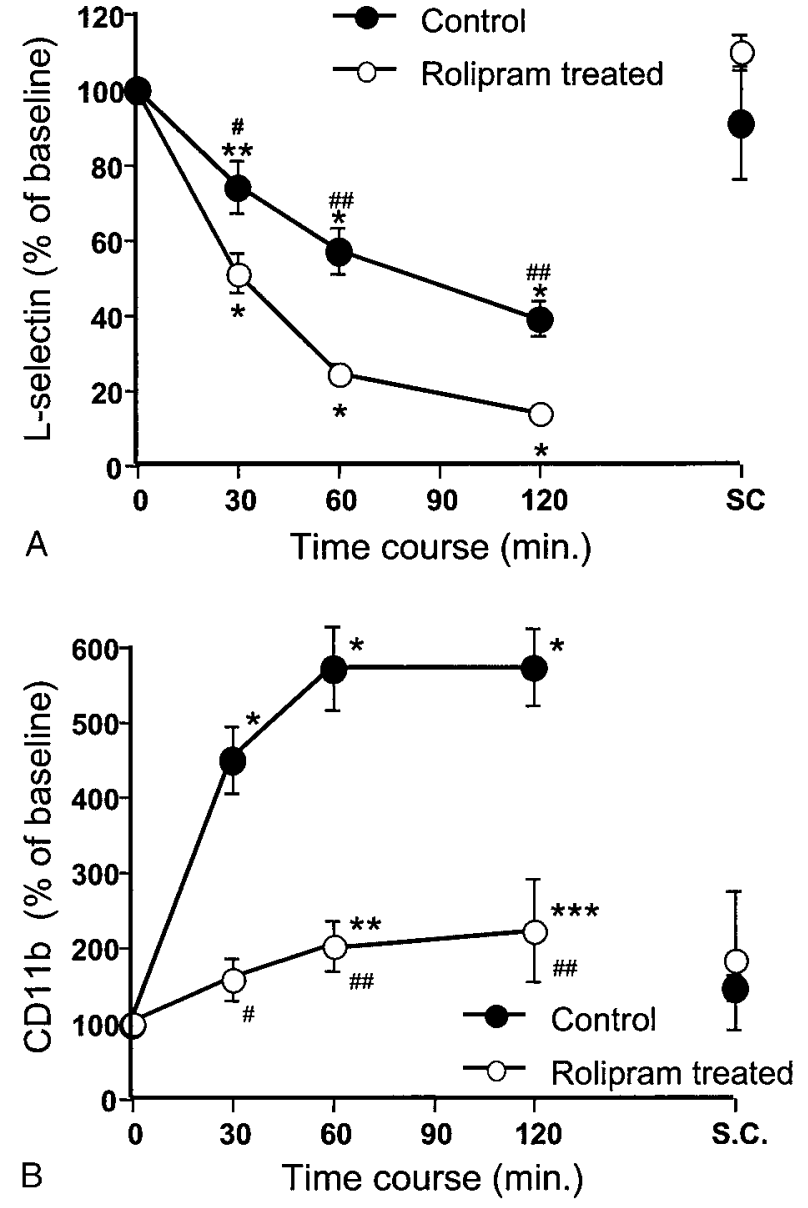

Figure 3. The expression of L-selectin and CD11b of PMNs. A, The expression of L-selectin of PMNs decreased in both the control and rolipram-treated groups. Rolipram attenuated this decrease. Results are expressed as means \pm SEM of 6 experiments. ${ }^{*} P<$ $.001,{ }^{*} P<.004$ versus baseline; $\# P=.042, \# P=.002$ versus the control group. $B$, The expression of CD11b of PMNs increased in both the control and rolipram-treated groups. Rolipram inhibited this increase. Results are expressed as means \pm SEM of 6 experiments. ${ }^{*} P<.001,{ }^{*} P=.014,{ }^{* * *} P=.034$ versus baseline; $\# P=.029, \# P=.003$ versus the control group. $S C$, Standing control.

simulates the size of capillaries. ${ }^{17}$ The passage of PMNs through these channels was visualized by using videomicroscopy (Figure 2, $A$ ), and the transit times of whole blood were compared on the basis of treatment (Figure 2, $B$ ). We have used this whole blood transit time as a surrogate marker for PMN deformability because PMNs are far less deformable compared with red blood cells. ${ }^{5}$ Aggregated platelets might also affect the transit time. However, there was no difference in platelet number and aggregation in response to adenosine diphosphate $(5-50 \mu \mathrm{mol} / \mathrm{L})$, as measured with a platelet aggregometer (model PAC-4S, NBS Hematracer, Japan), between the control and rolipram- 


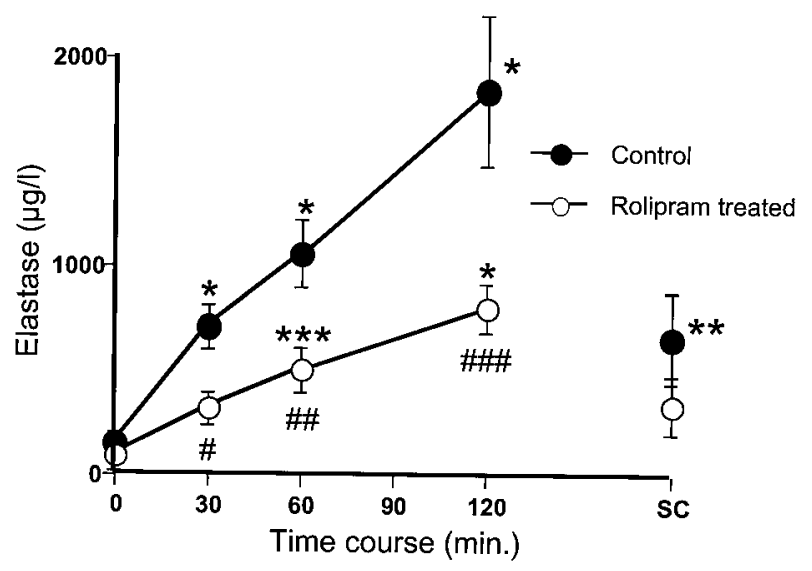

Figure 4. PMN elastase concentration. PMN elastase concentration increased in both the control and rolipram-treated groups. Rolipram attenuated this increase of PMN elastase concentration. Results are expressed as means \pm SEM of 6 experiments. ${ }^{*} \boldsymbol{P}<.001,{ }^{*} \boldsymbol{P}=.04,{ }^{*}{ }^{*} \boldsymbol{P}=.014$ versus baseline; $\# \boldsymbol{P}=.022$, $\# \#=.028, \# \# P=.042$ versus the control group. $S C$, Standing control.

treated groups during the experimental period (data not shown). Therefore, we suspect that PMN deformability change is the main factor that determines the transit time of whole blood through microchannels in our experiment. Because changes in the cytoskeleton with F-actin assembly at the cell periphery are thought to be responsible for the deformability change of PMNs, ${ }^{7,8}$ our results suggest that rolipram inhibits the deformability change of activated PMNs by inhibiting F-actin assembly. The deformability change of PMNs is important in initial sequestration of PMNs in microvessels. ${ }^{21}$ Therefore, rolipram is expected to inhibit initial PMN sequestration.

Simulated ECC decreased the L-selectin expression of PMNs (Figure 3, A). This is compatible with former studies with simulated ECC. ${ }^{22}$ On the other hand, CPB increases ${ }^{23}$ or does not alter ${ }^{24}$ the L-selectin expression of PMNs in clinical settings. We suspect that CPB causes the shedding of L-selectin from PMNs, which results in the decrease of L-selectin expression, but the release of L-selectin-rich PMNs from the bone marrow compensates for the change. ${ }^{25}$ Simulataed ECC increased the CD11b expression of PMNs (Figure 3,B), which is compatible with the results of former studies with simulated $\mathrm{ECC}^{22,26}$ and most clinical studies. ${ }^{24,27}$ The selectins slow PMNs by mediating rolling, and the integrins induce firm adhesion between PMNs and endothelial cells. ${ }^{6}$ These changes in the adhesive quality of PMNs are important in prolonged sequestration of PMNs in microvessels. ${ }^{9}$ Therefore, rolipram is expected to inhibit prolonged sequestration of PMNs in microvessels.

Simulated ECC induced the release of elastase from PMNs (Figure 4), and rolipram significantly inhibited this release. CPB induces the release of protease ${ }^{20}$ and reactive oxygen species ${ }^{2}$ from PMNs. The release of elastase and reactive oxygen species from PMNs sequestered in microvessels could cause damage to endothelial cells and induce tissue injury. ${ }^{2}$ There is a positive correlation between elastase plasma concentration during CPB and postoperative respiratory dysfunction. ${ }^{28}$ Our results suggest that rolipram could inhibit the endothelial injury caused by PMNs.

In summary, the results of this study show that the selective PDE type 4 inhibitor rolipram inhibits functional changes of PMNs in a model of simulated ECC and promotes their sequestration in microvessels. The sequestration of PMNs in microvessels is a crucial step in PMN-mediated organ injuries. Rolipram also inhibits the elastase release from PMNs, which causes endothelial injury. Therefore, this study suggests that PDE type 4 inhibition could be a future therapeutic strategy to prevent exaggerated inflammatory response and organ injuries related to $\mathrm{CPB}$, although further experimental work in animal models of CPB is necessary to determine the effects of PDE 4 inhibition in vivo.

We thank Dr Stephan F. van Eeden for reviewing the manuscript and Dr Yuji Kikuchi for direction in the measurement of PMN transit time through microchannels.

\section{References}

1. Johnson D, Thomson D, Hurst T, Prasad K, Wilson T, Murphy F, et al. Neutrophil-mediated acute lung injury after extracorporeal perfusion. J Thorac Cardiovasc Surg. 1994;107:1193-202.

2. Edmunds LH Jr. Inflammatory response to cardiopulmonary bypass. Ann Thorac Surg. 1998;66:S12-6; discussion S25-8.

3. Zimmerman GA, Amory DW. Transpulmonary polymorphonuclear leukocyte number after cardiopulmonary bypass. Am Rev Respir Dis. 1982;126:1097-8.

4. Kirklin JK, Westaby S, Blackstone EH, Kirklin JW, Chenoweth DE, Pacifico AD. Complement and the damaging effects of cardiopulmonary bypass. J Thorac Cardiovasc Surg. 1983;86:845-57.

5. Hogg JC, Doerschuk CM. Leukocyte traffic in the lung. Ann Rev Physiol. 1995;57:97-114.

6. Carlos TM, Harlan JM. Leukocyte-endothelial adhesion molecules. Blood. 1994;84:2068-101.

7. Frank RS. Time-dependent alterations in the deformability of human neutrophils in response to chemotactic activation. Blood. 1990;76: 2606-12.

8. Worthen GS, Schwab Bd, Elson EL, Downey GP. Mechanics of stimulated neutrophils: cell stiffening induces retention in capillaries. Science. 1989;245:183-6.

9. Doerschuk CM. The role of CD18-mediated adhesion in neutrophil sequestration induced by infusion of activated plasma in rabbits. Am J Respir Cell Mol Biol. 1992;7:140-8.

10. Torphy TJ. Phosphodiesterase isozymes: molecular targets for novel antiasthma agents. Am J Respir Crit Care Med. 1998;157:351-70.

11. Anderson R, Goolam Mahomed A, Theron AJ, Ramafi G, Feldman C. Effect of rolipram and dibutyryl cyclic AMP on resequestration of cytosolic calcium in FMLP-activated human neutrophils. $\mathrm{Br} J$ Pharmacol. 1998;124:547-55.

12. Sayeed MM. Exuberant $\mathrm{Ca} 2+$ signaling in neutrophils: a cause for concern. News Physiol Sci. 2000;15:130-5.

13. Hennessy VL, Hicks RE, Niewiarowski S, Edmunds LH, Colman RW. Function of human platelets during extracorporeal circulation. Am J Physiol. 1977;232:H622-8. 
14. Rinder CS, Rinder HM, Johnson K, Smith M, Lee DL, Tracey J, et al. Role of C3 cleavage in monocyte activation during extracorporeal circulation. Circulation. 1999;100:553-8.

15. Wachtfogel YT, Kucich U, Hack CE, Gluszko P, Niewiarowski S, Colman RW, et al. Aprotinin inhibits the contact, neutrophil, and platelet activation systems during simulated extracorporeal perfusion. J Thorac Cardiovasc Surg. 1993;106:1-9.

16. Sato Y, Hogg JC, English D, van Eeden SF. Endothelin-1 changes polymorphonuclear leukocytes' deformability and CD11b expression and promotes their retention in the lung. Am J Respir Cell Mol Biol. 2000;23:404-10.

17. Kikuchi Y. Effect of leukocytes and platelets on blood flow through a parallel array of microchannels: micro- and macroflow relation and rheological measures of leukocyte and platelet activities. Microvasc Res. 1995;50:288-300.

18. Holland BS, Copenhaver MD. An improved sequential rejective Bonferroni test procedure. Biometrics. 1987;42:417-23.

19. Chenoweth DE, Cooper SW, Hugli TE, Stewart RW, Blackstone EH, Kirklin JW. Complement activation during cardiopulmonary bypass: evidence for generation of $\mathrm{C} 3 \mathrm{a}$ and $\mathrm{C} 5 \mathrm{a}$ anaphylatoxins. $N \mathrm{Engl}$ J Med. 1981;304:497-503.

20. Wachtfogel YT, Kucich U, Greenplate J, Gluszko P, Abrams W, Weinbaum G, et al. Human neutrophil degranulation during extracorporeal circulation. Blood. 1987;69:324-30.

21. Doerschuk CM. Neutrophil rheology and transit through capillaries and sinusoids. Am J Respir Crit Care Med. 1999;159:1693-5.

22. Finn A, Morgan BP, Rebuck N, Klein N, Rogers CA, Hibbs M, et al. Effects of inhibition of complement activation using recombinant soluble complement receptor 1 on neutrophil CD11b/CD18 and Lselectin expression and release of interleukin- 8 and elastase in simulated cardiopulmonary bypass. J Thorac Cardiovasc Surg. 1996;111: 451-9.

23. McBride WT, Armstrong MA, Crockard AD, McMurray TJ, Rea JM. Cytokine balance and immunosuppressive changes at cardiac surgery: contrasting response between patients and isolated CPB circuits. Br J Anaesth. 1995;75:724-33.

24. Le Deist F, Menasche P, Kucharski C, Bel A, Piwnica A, Bloch G. Hypothermia during cardiopulmonary bypass delays but does not prevent neutrophil-endothelial cell adhesion. A clinical study. Circulation. 1995;92(Suppl):II-354-8.

25. Van Eeden S, Miyagashima R, Haley L, Hogg JC. L-selectin expression increases on peripheral blood polymorphonuclear leukocytes during active marrow release. Am J Respir Crit Care Med. 1995;151: 500-7.

26. Rinder CS, Rinder HM, Smith BR, Fitch JC, Smith MJ, Tracey JB, et al. Blockade of C5a and C5b-9 generation inhibits leukocyte and platelet activation during extracorporeal circulation. J Clin Invest. 1995;96:1564-72.

27. Rinder CS, Bonan JL, Rinder HM, Mathew J, Hines R, Smith BR. Cardiopulmonary bypass induces leukocyte-platelet adhesion. Blood. 1992;79:1201-5.

28. Hashimoto K, Miyamoto H, Suzuki K, Horikoshi S, Matsui M, Arai T, et al. Evidence of organ damage after cardiopulmonary bypass. The role of elastase and vasoactive mediators. J Thorac Cardiovasc Surg. 1992:104:666-73. 